\title{
LAS EMPRESAS DE INSERCIÓN EN ESPAÑA EN 2019
}

\author{
Miguel Ángel García Calavia \\ Profesor Titular de Universidad \\ Departamento de Sociología y Antropología Social \\ IUDESCOOP - Universitat de València
}

\section{RESUMEN}

En el presente artículo, se ofrecen los resultados de una radiografía que se ha llevado a cabo de las empresas sociales de integración laboral en España, cinco años después de dada por finalizada la gran recesión de este siglo (2009-2014). Se trata de iniciativas ya consolidadas que han evidenciado su capacidad de innovación en la búsqueda de soluciones adecuadas tanto en lo que se refiere al entorno territorial como a los distintos grupos destinatarios. Además, en la investigación empírica se quiere profundizar en una de las funciones sociales básicas de este tipo de empresas: la de intermediarias de transición de grupos sociales desfavorecidos en el mercado de trabajo.

Las fuentes de información para la radiografía provienen de documentación de FAEDEI y de entrevistas en profundidad a informantes cualificados, mediadores laborales de las WISE. Los resultados han permitido profundizar en el conocimiento de los procesos de inserción de las personas con serias dificultades para encontrar empleo y en riesgo de exclusión social.

PALABRAS CLAVE: Personas inempleables, exclusión social, mediación laboral, España.

CLAVES ECONLIT: A13, J49, P31.

Cómo citar este artículo/How to cite this article: GARCÍA CALAVIA, M.A.: “Las empresas de inserción en España en 2019", CIRIEC-España, Revista Jurídica de Economía Social y Cooperativa, $\mathrm{n}^{\circ}$ 36, 2020, pp. 131-152. DOI: 10.7203/CIRIEC-JUR.36.17307.

1. El presente artículo forma parte de los resultados del Proyecto Economía Social, Autogestión y Empleo (DER2016-78732-R), ECOSOCIAL 2020, financiado por el Ministerio de Ciencia, Innovación y Universidades y el Fondo Europeo de Desarrollo Regional. 


\section{WORK INTEGRATION SOCIAL ENTERPRISES (WISE) IN SPAIN IN 2019}

\section{EXPANDED ABSTRACT}

In this paper we present the first results of a service which is being carried out of Work Integration Social Enterprise (WISEs) specializing in Spain five years after the date which has been taken as the end of this century's economic crisis (2009-2014). It covers already consolidated initiatives which have been shown to be innovative in looking for adequate solutions for particular areas and target groups. In this description, attention is paid to a series of variables in order to assess the economic dynamics of this type of company. In addition, in the empirical research we have wanted to explore in more depth one of the social functions of these organizations: that of acting as intermediaries to facilitate the transition into the labour market of disadvantaged social groups.

The objectives are therefore descriptive. However, the examination allows evaluating the effectiveness of the Work Integration Social Enterprise as social companies. Also, investigate the exercise of one of its functions, that of labor mediation, carried out by someone from the insertion company. This is a very important responsibility, on the one hand, in the search for jobs outside WISE; on the other, in the training and orientation of insertion workers (WIs); finally, in its subsequent follow-up. However, the performance of this function is poorly understood. His examination is one of the contributions of this article.

In some case studies, the importance of training of a different nature provided in the WISEs with respect to the insertion of WIs in the ordinary market has been examined. It has been shown that personal and social skills are more important than professional skills, often very routine, in insertion. In this regard, it was urged to investigate the labor mediation process and its protagonists.

The sources of information for the survey are the documentation of Federación de Asociaciones Empresariales de Empresas de Inserción (FAEDEI) and a survey which is being carried out. The variables selected for the description are legal (legal formulas of the insertion companies), economic (activity sectors, templates and composition, income and origin thereof) and social (insertion results). In the narrations of the interviewees, attempts have been made have allowed us to delve into the attitudes of the protagonists of the insertion, WIs and ordinary companies, as well as those of the interviewed mediators themselves; in the contents of the insertion practices. 
The description has shown that insertion companies respect and meet the economic criteria to be considered social companies: they carry out productive activities over time; they constitute autonomous projects of the public administrations; its activities are market oriented; they involve business risk; and require a minimum of salaried workers. In 2018, WISE have been promoted mainly by non-profit entities created under different legal formulas; above all, under the Foundation or Association. The activities carried out by the WISEs are located mainly in the service sector: almost $80 \%$. They are labor intensive activities and little capital investment. Most WISE do more than one activity, often two, which are usually complementary. The income of the WISEs came mainly from the sale of products and services: almost $80 \%$ of the income; a percentage that has been maintained since 2010. In this respect, the WISEs transmit the will to be solvent, although they are not yet solvent. In addition, the number of WISE workers has increased considerably in recent years, especially that of WIs: between 2015 and 2018, 27.8\%. Regarding its distribution by sex, the number of female workers is quite similar to that of male workers.

Likewise, they satisfy one of the main social criteria: provide a service to the community, social integration through the work of people with difficult employability, contributing to social cohesion. In 2018 , almost $70 \%$ of the people who had completed their learning pathways in WISEs, entered the ordinary labor market. This percentage represented a considerable increase with respect to the insertion registered in 2015 and 2012. Insertion companies are, therefore, social companies.

On the other hand, the examination of the information contained in the in-depth interviews has allowed to deepen the knowledge of the insertion processes of people with serious difficulties in finding employment and at risk of social exclusion. The existence of people with job mediation functions in WISEs has been verified, as well as the availability of networks typical of ordinary companies to turn to when WIs are about to complete their learning itineraries and end their employment contracts. Regarding the insertion processes, it has been observed the importance of the attitude of the WIs in the conclusion of the learning itinerary and in the job search, the development of occupational training programs of the WIs in the search for employment and the existence of an informal mediation channel alongside the formal one. With regard to the immediately subsequent phase related to employment in ordinary companies, a significant degree of satisfaction from the contracted WIs, as well as from the contracting ordinary companies themselves.

Thus, the importance of mediators and their action in the transition from WIs to the ordinary labor market has been demonstrated, both in terms of prospecting for WIs and in their employment orientation. Also, the importance of the predisposition of the WIs in regards to 
completing the itineraries, as well as facing the insertion. They are the main contributions of this article.

Finally, some problems that affect the activity of the WISEs have been shown: the lack of visibility of their function, which hinders their political and social recognition; poor long-term coordination with public administrations to guarantee WISE activity; the absence of a unique regulation of conciliation for WIs who start their working life in ordinary employment so that they can better combine it with their personal and family life; the predominance of highly feminized productive activities in WISE, limiting the professional training of WIs.

KEYWORDS: Unemployed people, social exclusion, labor mediation, Spain. 


\section{SUMARIO}

1. Introducción. 2. Las empresas de inserción. Papel y problemas como agentes intermediarias de transición al mercado laboral. 3. Fuentes de información y metodología. 4. Forma jurídica de las EIs. 5. Distribución territorial de las EIs. 6. Los sectores y subsectores en los que desarrollan su actividad las EIs. 7. Ingresos económicos de las EIs y procedencia. 8. El personal de las EIs. 9. Resultados de inserción de las PTIs en el mercado laboral ordinario. 10. Los procesos de inserción. 11. Conclusiones. Bibliografía.

\section{Introducción}

En el presente artículo, se lleva a cabo una radiografía de las empresas de inserción (EI) en España, cinco años después de dada por finalizada la gran recesión (20092014). Se trata de empresas que ejercen una actividad productiva de manera autónoma y económicamente viable en su correspondiente mercado del producto y cuyo objetivo principal es la capacitación de personas de difícil empleabilidad mediante un empleo para que puedan ocupar posteriormente un puesto "ordinario" e integrarse en la sociedad (Marcuello, Bellostas y Marcuello, 2008); esto es, de personas en riesgo de exclusión social, una de cuyas razones reside en serios problemas para acceder a un puesto de trabajo ordinario. En esta radiografía, se presta atención a una serie de variables de carácter económico con el fin de valorar la dinámica económica de este tipo de empresas. Además, se examina algunas funciones con respecto al mercado de trabajo y la inclusión social; especial interés suscita su papel como intermediarias de transición de grupos sociales desfavorecidos en el mercado de trabajo. Los objetivos son, pues, de carácter descriptivo. Ahora bien, el examen permite valorar la operatividad de las EIs como empresas sociales y profundizar en el conocimiento de algunas de sus funciones.

Desde finales del siglo pasado, las políticas de empleo y protección social se han reorientado. Así, se han aprobado programas con el fin de abordar los problemas del mercado de trabajo y de inclusión social. Entre otros, algunos orientados a colectivos con especiales dificultades de inserción en el mercado de trabajo, entre los que se encuentran iniciativas empresariales diversas como la de las empresas de inserción por el trabajo. A este respecto, sus resultados avalan este tipo de programas (Faedei, 2018). En Espańa, este tipo de iniciativas que tienen un recorrido de casi 40 años, están reguladas desde 2007 por la Ley 44/2007. En la misma, se regula el funcionamiento de 
estas empresas, los destinatarios de los programas, su modo de acreditación, el tipo de relación laboral que se puede establecer o el tipo de acciones a realizar para alcanzar el objetivo de la inserción.

La estructura del artículo es la siguiente. En primer lugar, se caracteriza las empresas sociales para contextualizar mejor la radiografía de las EIs en España y se revisa la bibliografía sobre los papeles que desempeñan en el ámbito de la integración por el trabajo con el fin de extraer los problemas que han podido surgir en su ejercicio, especialmente, con respecto a su papel como agentes intermediarios. En segundo lugar, se comentan las fuentes de información. En tercer lugar, se radiografían las empresas de inserción en términos globales, su distribución territorial, sus actividades productivas, sus ingresos, sus plantillas de trabajadores y composición. En cuarto lugar, se examina los procesos de la fase final de estancia de las Personas Trabajadoras de Inserción (PTIs) orientados a la transición al mercado de trabajo ordinario observando el papel de sus protagonistas; también, los problemas que verbalizan. Para finalizar, se derivan algunas conclusiones con respecto a los objetivos señalados. Por un lado, la operatividad actual de las EIs. Por otro, la importancia de determinadas figuras, los mediadores laborales, en esta transición de PTIs al empleo ordinario.

\section{Las empresas de inserción. Papel y problemas como agentes intermediarias de transición al mercado laboral}

Las Empresas de Inserción surgieron inicialmente promovidas por colectivos procedentes de la sociedad civil organizada para afrontar la inserción laboral de personas con bajos o nulos niveles de empleabilidad en riesgo de exclusión social. Con este fin, adoptaron fórmulas del ordenamiento jurídico existente tendiendo a identificarse con las de la economía social. Estas iniciativas sociales encajaron pronto y bien en la nueva economía social y solidaria que se desarrolló a finales de siglo y que fue sistematizada a través de los conceptos de emprendimiento social y empresas sociales.

La perspectiva de análisis europea primó una caracterización de las empresas sociales a partir de la investigación empírica realizada en los primeros ańos del siglo. A este respecto, se distinguieron criterios económicos y sociales (Cuadro 1). Los criterios económicos permiten identificar su dinámica empresarial (en tanto que organizaciones orientadas a la producción de bienes y servicios), pero también apreciar su carácter innovador en el entorno del tercer sector. Los criterios sociales permiten ubicar a las empresas sociales igualmente en el tercer sector en tanto que sus objetivos (y prácticas para conseguirlo) son diferentes de las empresas tanto privadas como públicas. 


\section{Cuadro 1.}

\section{Criterios de las Empresas Sociales}

\begin{tabular}{|c|c|}
\hline Criterios económicos & Criterios sociales \\
\hline $\begin{array}{c}\text { Actividad continua de producción de } \\
\text { bienes y servicios }\end{array}$ & $\begin{array}{c}\text { Un objetivo explícito de servicio a una } \\
\text { comunidad o colectivo } \\
\text { Iniciativa ciudadana }\end{array}$ \\
(no tanto una actividad de defensa de & $\begin{array}{c}\text { (dinámica colectiva de personas que } \\
\text { pertenecen a una comunidad) } \\
\text { intereses o de redistribución de recursos } \\
\text { financieros) }\end{array}$ \\
Elevado grado de autonomía & $\begin{array}{c}\text { propiedad de capital } \\
\text { (decisiones asentadas en procesos de }\end{array}$ \\
(promovidos y gestionados por grupos & carácter democrático -una persona, un voto-) \\
de personas con autonomía frente a otras & Representación y participación democrática \\
organizaciones o los poderes públicos) & (implicando a los actores involucrados en \\
Significativo nivel de riesgo económico & su actividad) \\
(su viabilidad depende del esfuerzo y & Distribución limitada de beneficios \\
capacidad de sus miembros y trabajadores) & beneficio) \\
Empresas que orientan su actividad al & mercado \\
(una parte importante de sus ingresos & \\
proceden de sus ventas en el mercado) & \\
Mínimo de trabajadores remunerados & \\
(combinan recursos monetarios y no & monetarios)
\end{tabular}

Fuente: EMES.

Las EIs han sido asumidas como medidas de política activa de empleo e inclusión social (Vidal y Claver, 2003 y Quintao, 2007). Estas iniciativas han evidenciado su capacidad de innovación en la búsqueda de soluciones adecuadas, por un lado, a los diferentes entornos económicos y territoriales en los que se ubican y, por otro, a los distintos grupos destinatarios u objetivo de las mismas (Borzaga y Loss, 2006: 172). Así, las empresas de inserción han ejercido funciones de:

1. Exploradores de nichos de mercado y oportunidades de creación de empleo en sus respectivos territorios;

2. Promotores de competencias profesionales, así como, de sociabilidad, orientadas a la entrada en el mercado de trabajo y a la inclusión en la sociedad (...);

3. Agentes intermediarios de transición de grupos desfavorecidos en el mercado de trabajo;

4. Integradores de personas potencialmente productivas en entornos de sociabilidad, ocupación, formación y trabajo, desarrollando las adaptaciones necesarias. 
Un ejercicio de funciones que no ha sido siempre lineal ya que ha podido estar afectado por algunos hechos. Así, la de agentes de transición, por los tipos de actividad seleccionados por las EIs, de bajo valor ańadido y pocos recursos tecnológicos, que limitan la adquisición de competencias técnico profesionales (la cualificación) de los trabajadores en inserción y sus posibilidades de acceso posterior a otras actividades constrińnéndolos al segmento secundario del mercado laboral ordinario (Borzaga, Guí y Povinelli, 1997). Ahora bien, todavía se conoce poco la importancia (y el alcance) que esta circunstancia puede tener en el proceso mismo de la inserción, por otro lado, tampoco muy conocido, aunque cada vez más, sobre todo, en lo que se refiere a adquisición de competencias.

Recientemente se ha puesto de manifiesto, en algunos estudios de caso, que la capacitación técnico-profesional es muy elemental puesto que las tareas desempeñadas por los Trabajadores en Inserción son muy rutinarias y que se podría ampliar. Su aprendizaje reside en conseguir la velocidad y el ritmo adecuados para el ejercicio de las tareas, así como adquirir habilidades comunicativas para atenderlas y ejecutarlas. Así, la capacitación técnica profesional tiene poca trascendencia en su profesionalización, pero también a la hora de insertarse ocupacionalmente (una vez finalizado el contrato con las EIs): las competencias personales y sociales son más significativas que las competencias técnico profesionales. Por otro lado, se destaca la importancia de competencias transversales relacionadas con la búsqueda de empleo (Chisvert y al. 2018). A este respecto, se pone énfasis en la importancia de la mediación laboral que puede ser ejercida por alguna persona de la Empresa de Inserción compatibilizándola con alguna otra función o específicamente como prospector/a o mediador/a laboral en las empresas grandes.

En este contexto, se examina exploratoriamente este proceso de inserción en el mercado de trabajo ordinario (la fase final de la transición desde las empresas de inserción a las empresas ordinarias) mediante entrevistas en profundidad. Así, se examina si existen recursos humanos y procedimientos en las EIs con este fin en lo que se refiere tanto al contacto con empresas ordinarias, como a la provisión de competencias a las PTIs orientadas a la búsqueda de empleo y apoyo en su consecución. En segundo lugar, se examina las actitudes de los actores, esto es, empresas contratadoras, pero, asimismo, PTIs y, sobre todo, personal responsable de esta función en la EI. Con ello, se quiere contribuir a conocer el papel de las EIs como agentes intermediarios en el mercado laboral. Previamente, se observa la evolución de distintos aspectos de carácter económico de las EIs: sectores principales de actividad productiva de las EIs; plantillas y composición por sexo y tipo de personal; sus ingresos y su procedencia. Asimismo, resultados de inserción anuales. Su finalidad, es examinar la dinámica empresarial y social de las EIs y su ajuste a los criterios de las empresas sociales. 


\section{Fuentes de información y metodología}

Los datos de la radiografía provienen de las memorias sociales que anualmente redacta FAEDEI (Federación de Asociaciones Empresariales de Empresas de Inserción) a partir de la información que le proporcionan las EIs colaboradoras. Se trata de la principal fuente de información secundaria. Las variables seleccionadas son, en primer lugar, jurídicas: formas jurídicas de las EIs y de las entidades promotoras. En segundo lugar, económicas: sectores y ramas de actividad económica de las EIs, plantillas y composición, ingresos y procedencia de los mismos. En tercer lugar, sociales: resultados de la inserción. Para la radiografía, se utiliza la memoria más reciente, la de 2018, en la que colaboraron 185 EIs, un 78 \% de las EIs registradas. A efectos de observar su evolución, se ha seleccionado las memorias de 2015 y de 2018, último año del que hay memoria; y en relación a los resultados de inserción, se ha incluido también la de 2012 para poder tener como referencia los de un año del periodo de crisis.

Además, se han realizado entrevistas en profundidad a 6 personas responsables de la función de mediación en las EIs: 1 a una grande -aunque ahora ejerce la función en una agencia de colocación propia de la fundación promotora-; 2 (una mujer y un hombre) pertenecen a una empresa de tamaño mediano-grande; 2 a dos empresas de tamaño mediano pequeño -una con un programa específico para mujeres-; y 1 a una empresa pequeña. Así, están representados los distintos tipos de EI por tamaño y también, por recursos. Hay 5 mujeres y 1 hombre.

En las entrevistas en profundidad, se ha ahondado en las actitudes de las y los protagonistas de la inserción, PTIs y empresas ordinarias, así como en la de la propia persona responsable de la mediación en la EI; en los contenidos de las prácticas de inserción. Para ello, se ha solicitado a personas responsables de esta función en la EI que reconstruyeran los procesos de inserción lo que a su vez ha permitido derivar la filosofía bajo la que se promueve la inserción. La información contenida se ha clasificado conforme a los aspectos en los que se quería profundizar.

\section{Forma jurídica de las EIs}

La forma jurídica adoptada por las EIs de manera abrumadoramente mayoritaria es la de Sociedad Limitada: 4 de cada 5 EIs participantes en la memoria la han adoptado. También, hay unas pocas EIs que se han constituido bajo las fórmulas de sociedad laboral y sociedad cooperativa. Este predominio de la fórmula de sociedad limitada desde hace una década guarda relación con lo establecido en el artículo 4 
de la Ley 44/ 2007 en la que se regula el régimen de las Empresas de Inserción. En el mencionado artículo, se fija que las EIs se pueden constituir bajo las fórmulas de sociedad mercantil o sociedad cooperativa.

\section{Cuadro 2.}

Fórmulas jurídicas de las EIs en 2015 y 2018

\begin{tabular}{|l|c|c|}
\hline & $\mathbf{2 0 1 5}$ & $\mathbf{2 0 1 8}$ \\
\hline Sociedad limitada & 108 & 103 \\
\hline Sociedad laboral & 3 & 13 \\
\hline Sociedad Cooperativa & 9 & 9 \\
\hline Otras & 5 & 1 \\
\hline Total & 125 & 126 \\
\hline
\end{tabular}

Fuente: FAEDEI.

Esta primacía de la sociedad limitada es debida al bajo capital inicial requerido para su constitución ya que resulta más fácil su gestión. La razón contraria, esto es, una gestión más complicada ayuda a explicar el bajo recurso a las fórmulas de la economía social, las cooperativas y las sociedades laborales, entre otros hechos, por la transitoriedad de las personas trabajadoras de inserción.

Las EIs han sido promovidas a su vez por entidades sin ánimo de lucro o públicas fundadas bajo diferentes fórmulas jurídicas. Las entidades sin ánimo de lucro han recurrido a las de Fundación (60), Asociación (39) o Cooperativa (28).

\section{Distribución territorial de las EIs}

En lo que se refiere a la distribución geográfica de las EIs por comunidades autónomas, se pueden establecer varios grupos. En primer lugar, el constituido por Catalunya y País Vasco; concentran el 53 \% del total. En segundo lugar, el configurado por Aragón, Comunidad de Madrid y Castilla León; concentran, casi el 19 \%. Un tercer grupo, el constituido por Comunidad Valenciana, Navarra, Galicia, Castilla la Mancha, Asturias y Murcia. Luego, hay un último grupo, con una presencia muy limitada.

Esta desigual presencia guarda relación, en algún caso, País Vasco, con las dotaciones y ayudas proporcionadas por el gobierno vasco, pero probablemente, también, con el tipo de cultura social arraigada en el territorio: en el caso de los territorios del primer grupo, la economía social está más asumida que en el resto. 


\section{Los sectores y subsectores en los que desarrollan su actividad las EIs}

Según los datos recogidos en los Balances Sociales de FAEDEI, las actividades realizadas mayoritariamente por las EIs se ubican en el sector servicios: casi un $80 \%$ en 2018. Las actividades de las EIs restantes se localizan casi por igual en la agricultura, la construcción y la industria. Estos porcentajes apenas han cambiado en los últimos 4 años. En el cuadro 3, se recoge las actividades que con más frecuencia realizan las EIs. También, las actividades destacadas dentro del sector industrial que, aunque no son muchas las EIs que las llevan a cabo, sin embargo, son significativas de otro tipo de actividades.

\section{Cuadro 3.}

Ramas de actividad productiva más frecuentes a las que adscribieron las EIs sus actividades en 2015 y 2018

\begin{tabular}{|l|c|c|}
\hline & $\mathbf{2 0 1 5}$ & $\mathbf{2 0 1 8}$ \\
\cline { 2 - 3 } & Número & Número \\
\hline Agricultura y servicios relacionados con la misma (01) & 7 & 9 \\
\hline Industria textil (13) & 7 & 5 \\
\hline Confección de prendas de vestir (14) & 2 & 4 \\
\hline Edición, artes gráficas y reproducción (18) & 3 & 5 \\
\hline Recogida, tratamiento y eliminación de residuos (38) & 20 & 32 \\
\hline Construcción de edificios (42) & 6 & 7 \\
\hline Actividades de construcción especializada (43) & 7 & 4 \\
\hline Comercio al por menor (47) & 12 & 15 \\
\hline Servicios de comidas y bebidas (56) & 15 & 17 \\
\hline Servicios a edificios y actividades de jardinería (81) & 21 & 19 \\
\hline Actividades de servicios sociales sin alojamiento (88) & 12 & 10 \\
\hline Otros servicios personales (96) & 14 & 12 \\
\hline
\end{tabular}

Fuente: FAEDEI. ( ) Código CNAE.

Las actividades que han realizado más frecuentemente las EIs están relacionadas, en primer lugar, con "Recogida, tratamiento y eliminación de residuos", esto es, recogida de ropa, electrodomésticos, muebles... Su impacto social es doble: por un lado, laboral ya que permite desarrollar a las PTIs sus capacidades físicas y emocionales; por otro, medioambiental ya que posibilita reutilizar residuos. En segundo lugar, con "servicios a 
edificios y actividades de jardinería"; al menos, las actividades de jardinería pueden proporcionar una capacitación profesional singular. En tercer y cuarto lugar, servicios de comidas y bebidas; y comercio al por menor, una parte de las EIs se dedica al comercio justo. Otras actividades que efectúan asimismo numerosas EIs son otros servicios personales, como el lavado de prendas, y actividades de servicios sociales sin alojamiento, como los cuidados de personas en el hogar. En el ámbito de la industria, las actividades seleccionadas están relacionadas con el textil, confección y artes gráficas.

La mayoría de las EIs realizan más de una actividad, frecuentemente, dos, que suelen ser complementarias. Una expresión de diversificación productiva que constata su voluntad de crecimiento y consolidación. Por otro lado, son actividades intensivas en mano de obra y poca inversión de capital -con excepción de las industriales-. Esto resulta coherente con el objetivo social de las EIs, al menos de las medianas y grandes, disponer y ofrecer el máximo número de puestos de trabajo para la inserción laboral como vía para la inclusión social.

\section{Ingresos económicos de las EIs y procedencia}

En 2018, la venta de productos y servicios representaba casi el $80 \%$ de los ingresos de la EIs; un porcentaje que se mantiene desde hace más de una década. En este sentido, las EIs transmiten la voluntad de ser solventes, aunque todavía no lo son, todavía no son autosuficientes. Por ello, reciben asimismo ayudas públicas que representan un 18,1\%, así como, ayudas privadas (donaciones), un 1,4\%, y otros ingresos (derivados de inmovilizado), un 2,2 \%. De todas maneras, no es de una manera generalizada: hay empresas cuyos ingresos provienen de ayudas públicas en un porcentaje inferior y otras muchas que no reciben ningún tipo de ayuda.

\section{Cuadro 4.}

Ingresos económicos de las EIs en 2015 y 2018. Procedencia

\begin{tabular}{|l|r|r|r|r|}
\hline \multirow{2}{*}{} & \multicolumn{2}{|c|}{2015} & \multicolumn{2}{c|}{2018} \\
\cline { 2 - 5 } & \multicolumn{1}{|c|}{ Ingresos } & \multicolumn{1}{c|}{$\%$} & \multicolumn{1}{c|}{ Ingresos } & \multicolumn{1}{c|}{$\%$} \\
\hline Venta de productos y servicios & 84.609 .826 & 79,3 & 113.359 .698 & 78,3 \\
\hline Ayudas Públicas & 17.993 .742 & 16,7 & 26.293 .963 & 18,1 \\
\hline Ayudas Privadas & 647.114 & 0,6 & 1.990 .568 & 1,4 \\
\hline Otros ingresos de explotación & 3.455 .325 & 3,3 & 3.129 .408 & 2,2 \\
\hline Total & 106.706 .007 & & 144.773 .637 & \\
\hline
\end{tabular}

Fuente: FAEDEI. 
Si se comparan estos datos con los de 2015, se observa que han tenido lugar incrementos significativos en todas las partidas con excepción de las incluidas en "otros ingresos de explotación": 25,3\% en venta de productos y servicios; un 31\% en ayudas públicas y un $67,4 \%$ en ayudas privadas.

Así, las empresas de inserción son empresas que desarrollan una actividad económica continúa orientada claramente al mercado puesta de manifiesto en que han crecido sus ingresos por venta de bienes y servicios. Sin embargo, un parte todavía depende de las ayudas públicas que también han aumentado en los últimos 4 años.

\section{El personal de las EIs}

El número total de personas trabajadoras en las EIs durante el año 2018 era de 7.154 (3.424 mujeres y 3.730 hombres, esto es, 306 hombres más) de las cuáles el $60,1 \%$ eran personas trabajadoras en inserción (PTI). Esta distribución por sexos sobre el total de personas trabajadoras, $47,8 \%$ de mujeres y $52,2 \%$ de hombres, se desequilibra más cuando se examina entre el personal trabajador en inserción: las trabajadoras representan el 44,3\%. Por el contrario, entre el personal que no está en inserción, esto es, el personal de acompañamiento, producción, administración..., las trabajadoras representan casi el 53,8\%.

\section{Cuadro 5.}

Evolución del personal de las EIs entre 2015 y 2018

\begin{tabular}{|lc|c|c|c|c|}
\hline \multirow{2}{*}{} & \multicolumn{2}{|c|}{2015} & \multicolumn{2}{c|}{2018} \\
\cline { 3 - 6 } & & Número & $\%$ & Número & $\%$ \\
\hline Personal Trabajador en Inserción & & 3.117 & 55,6 & 4.299 & 60,1 \\
\hline & Hombres & 1.736 & & 2.342 & \\
\hline & Mujeres & 1.381 & & 1.957 & \\
\hline Personal Técnico y no inserción & & 2.484 & 44,4 & 2.855 & 39,9 \\
\hline & Hombres & 1.147 & & 1.388 & \\
\hline & Mujeres & 1.337 & & 1.467 & \\
\hline Total & & 5.601 & 100,0 & 7.154 & 100,0 \\
\hline & Hombres & 2.883 & & 3.730 & \\
\hline Mujeres & 2.718 & & 3.424 & \\
\hline
\end{tabular}

Fuente: FAEDEI. 
Por otro lado, hay que reseñar que desde 2015, el número de personas trabajadoras en inserción y de personal técnico, de estructura y producción, ha aumentado considerablemente, especialmente, el primero, el de personas trabajadoras en inserción, un 27,8 \% entre 2015 y 2018. También, en los años anteriores tuvo lugar, salvo 2012. Si examinamos su evolución por sexo: se ha incrementado porcentualmente el número de mujeres.

En lo que respecta al personal técnico, de administración -(no inserción)-, ha aumentado también, aunque menos que el de personal trabajador de inserción, 13\%; más entre los hombres que entre las mujeres.

Así pues, la composición de las plantillas por sexo está bastante equilibrada. A este respecto, las EIs contribuyen a la corrección de la desigualdad social y, por tanto, a la mejora del bienestar y d la inclusión social.

Tanto un tipo de personal como otro tiene formalizada contractualmente la relación laboral. La mayor parte del personal técnico mediante modalidades indefinidas y la mayor parte del personal trabajador de inserción (casi un $90 \%$ ) mediante las modalidades propuestas por la Ley 44/2007.

\section{Resultados de inserción de las PTIs en el mercado laboral ordinario}

Según la legislación vigente, las personas trabajadoras de inserción pueden permanecer contratadas en una EI entre un mínimo de 6 meses y un máximo de 36 siguiendo un itinerario individualizado de inserción. Por tanto, todos los años hay un número de PTIs que finalizan sus contratos y que debieran acceder al mercado de trabajo ordinario frecuentemente con el apoyo de la EI en la que ha estado trabajando y adquiriendo las competencias necesarias de todo tipo. Ahora bien, hay asimismo un número de PTIs que abandonan el itinerario de inserción antes de haberse capacitado. Antes de ofrecer y examinar los resultados anuales de inserción y su evolución, se comenta el movimiento laboral de las PTIs en las EIs.

Tres hechos llaman la atención (Cuadro 6), en primer lugar, que el número de participantes ha crecido significativamente en los últimos tiempos: un $29 \%$ con respecto a 2015 y un $44 \%$, a 2012. En segundo lugar, la existencia de un grupo de PTIs que abandona el programa y que ha aumentado desde 2012. En tercer lugar, que se ha incrementado el número de personas que finaliza el contrato sin finalizar el itinerario de inserción. 


\section{Cuadro 6.}

Movimiento laboral de las PTIs en las EIs durante los años 2012, 2015 y 2018

\begin{tabular}{|l|r|r|r|r|r|r|}
\hline \multirow{2}{*}{} & \multicolumn{2}{|c|}{2012} & \multicolumn{2}{|c|}{2015} & \multicolumn{2}{|c|}{2018} \\
\cline { 2 - 7 } & \multicolumn{1}{|c}{ No } & \multicolumn{1}{|c|}{$\%$} & \multicolumn{1}{c|}{ No } & \multicolumn{1}{c|}{$\%$} & \multicolumn{1}{c|}{ No } & \multicolumn{1}{c|}{$\%$} \\
\hline PTI que abandona itinerario inserción & 156 & 6,5 & 253 & 8,1 & 431 & 10,0 \\
\hline PTI que continua itinerario inserción & 1.470 & 61,2 & 1.856 & 59,5 & 2.627 & 61,1 \\
\hline PTI que finaliza itinerario inserción & 645 & 26,9 & 761 & 24,5 & 962 & 22,4 \\
\hline PTI que finaliza contrato & 129 & 5,4 & 247 & 7,9 & 279 & 6,5 \\
\hline & 2.400 & 100,0 & 3.117 & 100,0 & 4.299 & 100,0 \\
\hline
\end{tabular}

Fuente: FAEDEI.

\section{Cuadro 7.}

Resultados de inserción laboral de PTIs durante 2012, 2015 y 2018

\begin{tabular}{|l|r|r|r|r|r|r|}
\hline \multirow{2}{*}{} & \multicolumn{2}{|c|}{2012} & \multicolumn{2}{c|}{2015} & \multicolumn{2}{c|}{2018} \\
\cline { 2 - 7 } & \multicolumn{1}{|c|}{ No } & \multicolumn{1}{c|}{$\%$} & \multicolumn{1}{c|}{ No } & \multicolumn{1}{c|}{$\%$} & \multicolumn{1}{c|}{ No } & \multicolumn{1}{c|}{$\%$} \\
\hline PTIs que se inserta como asalariado & & 34,8 & 323 & 42,4 & 520 & 54,0 \\
\hline PTIs que se inserta en la propia EIL & - & 2,3 & 22 & 2,9 & 121 & 12,6 \\
\hline PTIs que se inserta mercado como autónomo & - & 4,9 & 54 & 7,1 & 27 & 2,8 \\
\hline \multicolumn{1}{|c|}{ PTIs totales que se han insertado } & & 42,0 & 399 & 52,4 & 668 & 69,4 \\
\hline PTIs que pasan a situación de desempleo & - & 58,0 & 362 & 47,6 & 294 & 30,6 \\
\hline Total & - & 100,0 & 761 & 100,0 & 962 & 100,0 \\
\hline
\end{tabular}

Fuente: FAEDEI.

En lo que se refiere a la inserción de las PTIs en el mercado laboral ordinario (Cuadro 7), esto es, en un puesto de trabajo ordinario de una empresa normal, como hemos comentado, el número de personas insertadas tras finalizar sus itinerarios en 2018 fue de 668: 520 como trabajador por cuenta ajena; 121 en la propia EI; y 27 como trabajador por cuenta propia. Por tanto, se ha insertado casi un $70 \%$. Si se compara estos datos con los de años anteriores, se observa que han aumentado considerablemente: 17 \% en relación a los del año 2015; un 27,4 a los del año 2012; sobre todo, en las empresas ordinarias. Ahora bien, se desconoce cuáles son las razones que las explican. La menor inserción durante la crisis se explica no solo por el momento que atraviesan las empresas ordinarias sino también por una mayor competencia con los parados ordinarios. Por otro lado, las modalidades contractuales con las que se inserta las PTIs son temporales lo que se corresponde con la excesiva precarización de una parte del mercado laboral español. 


\section{Los procesos de inserción}

Los relatos contenidos en las entrevistas en profundidad permiten conocer los procesos que se desarrollan en las EIs, sobre todo, en la fase final de las trayectorias de las PTIs con el fin de facilitar y guiar su inserción en las empresas ordinarias, así como velar sus primeros pasos en las mismas.

Se hace referencia a diversidad de perfiles de las PTIs y, por tanto, variedad de actitudes durante su estancia en las EIs lo que nos ayuda a entender mejor los éxitos $y$, en este caso, fracasos de la inserción. Se trata de una empresa grande en la que trabajan casi dos decenas de PTIs con unos resultados de inserción anuales próximos al $50 \%$, esto es, más o menos la mitad de las personas que finalizan sus contratos se incorporan en empresas ordinarias. Se invoca la existencia de absentismo: "hay personas trabajadoras de inserción que no finalizan el itinerario, pero, no, de 3 años, ni tan siquiera de $2 \ldots$ sus ausencias son tan reiteradas que no realizan el itinerario ni se les puede acompañar. Y llegado el momento de la renovación al iniciarse el $2^{\circ}$ o el 3er año, no se les renueva" $(\mathrm{E} 1)^{2}$. Esto ayuda a entender lo que las estadísticas denominan abandono de los itinerarios de inserción.

Asimismo, se alude a que las personas no siempre quieren insertarse a la finalización del contrato en la EI. Tiene lugar en la empresa anterior y en otra empresa mediana grande con resultados de inserción semejantes (50\%): "Hay quienes prefieren aprovechar las prestaciones que les corresponden y volver a casa hasta unas semanas antes que se reduzcan o se acaben y volver activarse (E2)3; "algunas PTIs, al finalizar los 3 años, deciden retirarse a sus casas: ‘ $¡$ calmaj, no es mi momento ${ }^{\prime}$ he desatendido mi hogar y ahora tengo un ańo de prestación'. Pero esta actitud cambia antes de llegar al sexto mes, cuando la cantidad de la prestación se reduce. Entonces, se plantean la inserción con insistencia, urgencia...” (E1). Estas actitudes contribuyen a explicar también los fracasos de las EIs en la inserción inmediata.

Ahora bien, hay otra parte de PTIs, como evidencian los resultados de inserción, que asume que el contrato está finalizando y acepta que hay que buscar empleo, aunque no siempre con tranquilidad y seguridad. Se encuentra en la empresa grande: "algunos se inquietan: 'se está acabando mi zona de confort y no sé si he aprovechado suficientemente las oportunidades ofrecidas; tampoco sé si sabré hacer otra cosa diferente a la que he hecho hasta ahora' y confían que las personas de la EI o la Fundación, les proteja encontrándole otro trabajo" (E1). 
Esta fase final de la estancia de las PTIs en las EIs orientada a su transición a la empresa ordinaria comienza cuando han adquirido las competencias previstas (80 $\%$ ) en sesiones individuales y colectivas (talleres) de formación para proporcionar competencias de búsqueda de empleo, información de recursos de empleo... Se efectúa en todas las EIs medianas o grandes de las personas entrevistadas. A este respecto, se les ayuda a inscribirse en portales de empleo porque sus habilidades como usuarias de la red suelen ser bajas. También, se organiza el proceso de inserción confeccionando CVs con las PTIs, proveyendo listados de empresas, preparando entrevistas...

Las inserciones, en caso de producirse, no siempre tienen lugar en una empresa que proporcionan las responsables de mediación. A veces, conscientes de las limitaciones de las vías tradicionales, animan a las PTIs a explorar su red de contactos por limitados que sean: "tienes que irte abriendo paso en tu comunidad, ya sea en la asociación de inmigrantes, en el gimnasio, en la iglesia, en la asociación de madres y padres..." (E1). Esto comporta una ampliación de las vías de inserción, por otro lado, como sucede en el mercado laboral ordinario y de lo que son conscientes las personas responsables. En este entorno, subyace una concepción de la inserción basada en la búsqueda activa de empleo.

A pesar de sus reservas en relación a la eficiencia de la intermediación laboral formal derivada de la información disponible y de sus experiencias, las entrevistadas aluden a su articulación y funcionamiento con interés.

Así, una de ellas, trabajadora de acompañamiento en una empresa mediana/pequeña, ha compatibilizado esta actividad con el establecimiento de contactos con empresas del tejido productivo local con el fin de explorar la existencia de ofertas de empleos a los que poder remitir alguno de las PTIs que finalizaban sus contratos: "dado que el volumen de trabajadores que hay que insertar es pequeño... resulta suficiente que nosotras conozcamos un poco el entramado empresarial local y seamos capaces de generar unos mínimos contactos" (E6)

La entrevistada de la empresa grande promovida por Caritas que ha ejercido específicamente funciones de prospección empresarial, orientación y mediación laboral, pone de manifiesto en su relato que dichas funciones están formalizadas en su empresa: "buscar el encaje de las PTIs en el entramado empresarial local o más próximo, lo que requiere conocer... esa fuente potencial de trabajadores a los que hay que colocar". Recientemente, ha pasado a formar parte de la Agencia de Colocación que ha creado Caritas para atender las necesidades de inserción de las EIs que ha promovido en la ciudad, así como otros programas de inserción: "El contacto con las empresas cuando las personas a insertar son numerosas... tenía que estar regularizado. Fue el 
momento (2018) que Caritas eligió para dar este paso". Esta iniciativa la ha promovido en bastantes ciudades de España en las que desarrolla esta labor de inserción laboral y social.

Con la creación de las Agencias de Colocación, tiene lugar una división del trabajo de mediación: orientación, prospección e intermediación. Asimismo, el alcance de cada una de estas funciones es amplísimo. En lo que se refiere a prospección, se destaca que hay empresas fidelizadas ya que han repetido todos los años de existencia de la Agencia. Ahora bien, reconoce que la oferta de trabajo no siempre llega en el momento de salida del trabajador. De todas maneras, hacen referencia a que una parte de las empresas ordinarias les cuesta contratar PTIs: “... las modalidades de contratación incentivadas requieren una duración mínima de 6 meses lo que no es frecuente en determinadas actividades en el primer contrato"(E5)

Además de la labor de mediación laboral, se lleva a cabo una labor de seguimiento durante 12 meses: "durante la vigencia del contrato (en la empresa ordinaria) supervisamos el cumplimiento de sus condiciones, su salario, su jornada..." (E4) Asimismo, se realiza y se valora el grado de satisfacción de las partes, del trabajador y de la empresa, procurando alargar al máximo el contrato. A este respecto, se ofrece asesoramiento a las empresas sobre posibles bonificaciones que pueden recibir si se alargan los contratos. El balance que efectúa la entrevistada sobre el grado de cumplimiento de las empresas de las condiciones de los contratos es positivo: "sólo ha habido 2 empresas con las que hemos tenido que dejar de colaborar, porque estaban incumpliendo lo pactado. Había unas condiciones indignas de trabajo" (E1). Asimismo, suele haber pocas quejas empresariales respecto al trabajo que realizan las PTIs en las empresas ordinarias.

Cuando las PTIs disponen de las competencias mínimas, una de las personas entrevistadas de la empresa mediana grande inicia su formación para la búsqueda activa de empleo: elaboración de CV, redacción de carta de presentación, simulación de entrevistas...; asimismo, para que comiencen a ser autónomos: “...dejando atrás la idea de que Caritas no me abandonará conociendo mis condiciones de fragilidad" (E2). La inserción de las PTIs tiene dificultades, sobre todo, en un mercado laboral como el español donde existe una tasa de paro elevada y por tanto, competidores para ocupar un puesto de trabajo. Estas dificultades se agrandan en periodos de crisis. Una parte de las PTIs son conscientes de sus dificultades de inserción. Más aún, hay quienes consideran que plasmar en un CV o expresar en una entrevista que han pasado por una EI les puede perjudicar porque comporta manifestar la situación

5. E5: Responsable de empresa mediana pequeña dedicada a servicios de limpieza.

6. E4: Trabajadora de empresa mediana pequeńa dedicada a recogida y venta de ropa. 
anterior que han tenido: "prefieren que no aparezca para que no se indague en sus antecedentes (E2)".

Los relatos de las entrevistadas han permitido, pues, conocer mejor esta etapa final orientada a la inserción de las PTIs en la empresa ordinaria. En relación con sus estructuras y sus protagonistas, sus recursos:

1. Existencia de técnicas o técnicos con funciones de intermediación en las EIs. Incluso, existencia de agencias de colocación en el caso de las EIs grandes.

2. Disposición de redes propias de empresas ordinarias construidas a través de visitas para establecer convenios con las mismas una vez explicada la finalidad de una EI e informada la empresa ordinaria de las posibles ayudas que pueden recibir por contratación de PTI. En el desarrollo de este tipo de actividad cuentan con el apoyo y ayuda de programas sociales específicos de determinadas entidades privadas.

Con respecto a los procesos de inserción:

1. Desarrollo de programas de capacitación ocupacional de las PTIs en la búsqueda de empleo (en las que se enseña a preparar entrevista laboral y se proporcionan recursos de búsqueda de empleo -portales de empleo...-) a cargo de las Técnicas o Técnicos responsables de inserción.

2. Relevancia de la actitud de las PTIs en la finalización del itinerario de aprendizaje y, por tanto, que pueda disponer de las competencias necesarias para que la EI medie en su inserción en el mercado ordinario.

3. Existencia de una vía de mediación informal junto a la vía formal.

Por último, en relación con la etapa posterior a la salida de la PTI de la EI, un importante grado de satisfacción de la PTI, así como de las empresas ordinarias contratantes.

\section{Conclusiones}

Uno de los objetivos del presente artículo era radiografiar las empresas de inserción cinco años después de dada por finalizada la recesión económica de 2008-2014. Además, se quería observar si satisfacían los criterios establecidos como empresas sociales casi 20 años después de su formulación. 
En principio, la radiografía efectuada a partir de los datos contenidos en las memorias de FAEDEI ha puesto de manifiesto que las empresas de inserción son empresas que desarrollan actividades de bienes y servicios de distinto tipo a lo largo del tiempo. Han sido creadas voluntariamente por grupos de personas bajo distintas fórmulas en tanto que proyectos autónomos de las administraciones públicas u otras organizaciones. La mayor parte de sus ingresos proceden de la venta de sus bienes o servicios en el mercado (hasta el 80\%), por tanto, son empresas que orientan su actividad al mercado, aunque puedan depender de donaciones o subvenciones públicas (hasta un 20\%). A este respecto, el grupo promotor asume totalmente el riesgo empresarial que comporta la gestión diaria de la empresa de inserción y su viabilidad depende del esfuerzo de sus miembros y de los trabajadores que participan en el proyecto. Por último, traducen la existencia de un mínimo de trabajadores que ha aumentado en todo este tiempo. Así, cumplen los criterios de carácter económico.

Por otro lado, la actividad empresarial que realizan las EIs permite llevar adelante un objetivo explícito de servicio a la comunidad: la inserción por el trabajo de personas de difícil empleabilidad cuyos resultados han mejorado en los últimos años. En este sentido, benefician a este tipo de colectivos, pero asimismo a las comunidades contribuyendo a su cohesión social. Al mismo tiempo, facilitan la labor emprendida por las administraciones públicas para amortiguar la exclusión social.

En este contexto, las EIs son claramente empresas sociales que satisfacen no solo los criterios de cualquier empresa sino asimismo su objetivo principal de capacitar personas de difícil empleabilidad que se han ido incorporando de manera creciente al mercado ordinario. En la consecución de este objetivo, se ha puesto de manifiesto la importancia de la existencia de personas responsabilizadas de la función de mediación con respecto al mercado laboral en las EIs y de su acción. En primer lugar, son las personas responsables de crear redes de empresas o, al menos, contactar con empresas susceptibles de contratar a las PTIs, si bien este proceso puede encontrar dificultades: oferta y demanda no siempre coinciden en el tiempo; las modalidades contractuales incentivadas para la contratación de PTIs tienen una duración (6 meses) que supera la de las que son frecuentes en primeros contratos de actividades que son susceptibles de acogerlas. Esta actividad de prospección puede estar muy formalizada en algunas de las EIs grandes.

En segundo lugar, las personas responsables de la mediación en relación con la transición de las PTIs al empleo ordinario son las personas encargadas de su formación y orientación en la búsqueda de empleo. A este respecto, promueven y llevan a cabo las actividades propias de esta fase de manera colectiva e individual proporcionando recursos de distinto tipo a las PTIs. En tercer lugar, median en su inserción proporcionando empresas en las que existen demandas de trabajo a las que pueden 
presentarse, pero también proponiendo a las PTIs su activación mediante su inscripción en servicios o portales de empleo o a través de la exploración sus redes personales. Finalmente, son las responsables de hacer su seguimiento en el caso de que se inserten.

Las personas responsables de la mediación laboral en las EIs son, pues, actores centrales en la inserción tal como se planteaba en las conclusiones de algunos estudios de caso (Chisvert y al., 2018). Son un eslabón muy importante, por un lado, en la búsqueda de puestos de trabajo fuera de la ESIL; por otro, en la formación y orientación de las PTIs; por último, en el seguimiento posterior de la PTI.

Por otro lado, en sus relatos hacen referencia a determinadas anomalías que ayudan a entender los resultados de inserción. Asimismo, a determinados problemas que permitiría mejorar la actividad de las EIs. Entre las primeras, absentismo laboral de PTIs que imposibilita desarrollar itinerario de inserción o decisiones personales de PTIs de iniciar la búsqueda de empleo unos meses después de finalizada la relación contractual con la ESIL ya que tienen cubiertos unos meses mediante prestaciones por desempleo.

Entre los problemas, en primer lugar, la falta de visibilidad del papel de las EIs en la inclusión social a partir de los buenos resultados en la inserción laboral de personas con dificultades de empleabilidad. Esto dificulta su reconocimiento tanto por las administraciones públicas como por los actores sociales, especialmente, por las empresas ordinarias que han de contratar y, por tanto, una mejor intervención y acción. En segundo lugar, una mejor coordinación con las administraciones públicas a través de programas plurianuales para garantizar la actividad de las EIs con mayor estabilidad y eficiencia en los resultados de los programas. En tercer lugar, la ausencia de una regulación singular de la conciliación de las personas en situación de vulnerabilidad para que puedan compatibilizar mejor su vida personal y/o familiar con los requisitos de empleabilidad de las empresas ordinarias. Por último, el predominio de actividades productivas en las EIs asociadas a sectores productivos feminizados o adscritos a los roles tradicionales de género. A este respecto, la necesidad de diversificar más sus actividades productivas teniendo en cuenta sectores más estratégicos asumibles en las EIs, como, por ejemplo, las energías renovables. Este reconocimiento y esta propuesta constituyen una respuesta parcial que constata lo poco que se ha avanzado en la resolución del problema planteado por Borzaga, Povinelli y Gui a finales de siglo. 
(pp. 131-152)

\section{Bibliografía}

BORZAGA, C., GUI, B. \& POVINELLI, F.: "Le rôle des entreprises d'insertion sur le marché du travail - l'eclairage d'une analyse economique". En Insertion et nouvelle économie sociale (VV.AA.), Editions Desclée de Brouwer, Paris, 1997, pp. 267-291.

BORZAGA, C. \& LOSS, M.: "Profiles and trajectories of workers in work integration social enterprises". En Social Enterprise at the crossroads of market, public policies and civil society (Ed. NYSSENS, M.), Routledge, Londres, 2006, pp. 169174.

CHISVERT-TARAZONA, M.J., PALOMARES, D., HERNAIZ-AGREDA, N. et al.: "Trayectorias de aprendizaje en contextos informales. El caso de tres empresas de inserción”, CIRIEC-España, Revista de Economía Pública, Social y Cooperativa, no 94, 2018, pp. 185-216.

FAEDEI: Memoria social. Empresas de Inserción, Madrid, 2018.

MARCUELLO, C., BELLOSTAS, A. \& MARCUELLO, CH.: Informe sobre las empresas de inserción en España, CIRIEC-España, Valencia, 2008.

QUINTAO, C.: "Empresas de inserción y empresas sociales en Europa", CIRIECEspaña. Revista de Economía Pública, Socialy Cooperativa, no 59, 2007, pp. 33-59.

VIDAL, I. \& CLAVER, N.: "Las empresas sociales en el ámbito de la integración por el trabajo", CIRIEC-España, Revista de Economía Pública, Social y Cooperati$v a, \mathrm{n}^{\circ} 46,2003$, pp. $39-62$. 